\title{
A convenient access to new pyrido[4,3-d]pyrimidine, thiazolo[3,4-c]pyrimidine and pyrimido[4,5-d]pyridazine derivatives
}

\author{
Nabila A. Kheder, ${ }^{\text {a }}$ Yahia N. Mabkhot, ${ }^{b}$ and Ahmad M. Farag ${ }^{a_{*}}$ \\ ${ }^{a}$ Department of Chemistry, Faculty of Science, Cairo University, Giza 12613, Egypt \\ ${ }^{b}$ Department of Chemistry, Faculty of Science, King Saud University, 2455, Riyadh 11751, Saudi \\ Arabia \\ E-mail: afarag49@yahoo.com
}

\begin{abstract}
Several new pyrido[4,3- $d]$ pyrimidine, pyrimido[4,5- $d]$ pyridazine and thiazolo[3,4-c]pyrimidine derivatives were prepared from the versatile, readily accessible ethyl 6-methyl-2-oxo-4-phenyl1,2,3,4-tetrahydropyrimidine-5-carboxylate. Bromination of the latter compound afforded the corresponding 6-bromomethylpyrimidine derivative. Treatment of the latter with malononitrile or ethyl cyanoacetate afforded the corresponding hexahydrocyclopenta $[d]$ pyrimidine derivatives 4a,b. Reaction of the bromomethylpyrimidine with potassium cyanide followed by treatment with arenediazonium salt afforded the corresponding hydrazone 6 which reacts with hydrazine derivatives to afford the corresponding pyrido[4,3- $d]$ pyrimidine derivatives $\mathbf{8 a , b}$. It reacts also with thiourea, thiosemicarbazide and phenylhydrazine to afford ethyl 3-imino-5-oxo-7-phenyl3,5,6,7-tetrahydro-1- $H$-thiazolo[4,3-c]pyrimidine-8-carboxylate (10a), ethyl 3-hydrazono-5-oxo7-phenyl-3,5,6,7-tetrahydro-1- $H$-thiazolo[3,4-c]pyrimidine-8-carboxylate $\quad(\mathbf{1 0 b}) \quad$ and 4,6diphenyl-3,4,7,8-tetrahydropyrimido[4,5- $d$ ]pyridazine-2,5-(1H,6H)-dione (12), respectively. The antimicrobial activity of selected examples of the synthesized compounds was tested and showed moderate activity.
\end{abstract}

Keywords: 1,2,3,4-tetrahydropyrimidine, hexahydrocyclopenta[d]pyrimidine, pyrido[4,3$d]$ pyrimidine, thiazolo[3,4-c]pyrimidine, pyrimido $[4,5-d]$ pyridazine

\section{Introduction}

The pyrimidine nucleus is present in a wide range of bioactive natural products. In addition, the pharmacological and biological activities of pyrimidine derivatives are well documented. ${ }^{1-6}$ Encouraged by these findings and as a continuation of our interest in the synthesis of a variety of heterocyclic systems for biological screening, ${ }^{7-17}$ we have found that 6-bromomethylpyrimidine $\mathbf{2}^{18}$ is a versatile, readily accessible building block for the synthesis of new 
cyclopenta $[d]$ pyrimidne, pyrido[4,3- $d]$ pyrimidine, thiazolo[3,4-c]pyrimidine and pyrimido[4,5$d$ ]pyridazine derivatives of expected biological potency. Our interest in the above point prompted us to react compound $\mathbf{2}$ with some nucleophiles.

\section{Results and Discussion}

The versatile synthon ethyl 6-(bromomethyl)-2-oxo-4-phenyl-1,2,3,4-tetrahydropyrimidine-5carboxylate (2), ${ }^{18}$ was obtained via bromination of ethyl 6-methyl-2-oxo-4-phenyl-1,2,3,4tetrahydropyrimidine-5-carboxylate $(\mathbf{1})^{19}$ in acetic acid. Treatment of the compound $\mathbf{2}$ with malononitrile or with ethyl cyanoacetate afforded the corresponding hexahydrocyclopenta[ $[d]$ pyrimidne derivatives $\mathbf{4 a}$ and $\mathbf{4 b}$, respectively (Scheme 1).

This reaction is assumed to proceed via nucleophilic attack of a carbanion-methylene group, in malononitrile or ethyl cyanoacetate, on the 6-bromomethyl group in $\mathbf{2}$ to afford the nonisolable intermediates 3a,b which underwent intramolecular cyclization through elimination of ethanol to afford the final products $\mathbf{4 a , b}$. The structure of the products $\mathbf{4 a}, \mathbf{b}$ was determined from spectroscopic as well as elemental analytical data. Thus, compound 4a, taken as a typical example, showed absorption bands at 1650, 1696, 2195, 2210, 3102, $3350 \mathrm{~cm}^{-1}$ corresponding to two $\mathrm{C}=\mathrm{O}$ groups, two $\mathrm{C} \equiv \mathrm{N}$ and two $\mathrm{NH}$ functions, respectively. Its ${ }^{1} \mathrm{H}$ NMR spectrum revealed the absence of $\mathrm{CH}_{3}$ and $\mathrm{CH}_{2}$ protons of ethoxycarbonyl group and showed signals at $\delta 4.82$ and 5.25 due to $\mathrm{CH}_{2}, \mathrm{CH}$ protons, respectively. It showed also two $\mathrm{D}_{2} \mathrm{O}$-exchangeable signals at $\delta$ 7.75 and $9.97 \mathrm{ppm}$ corresponding to two NH protons, in addition to an aromatic multiplet in the region $\delta$ 7.26-7.39.<smiles>CCOC(=O)C1=C(C)NC(=O)NC1c1ccccc1</smiles>

1<smiles>CCOC(=O)C1=C(CBr)NC(=O)NC1c1ccccc1</smiles>

2

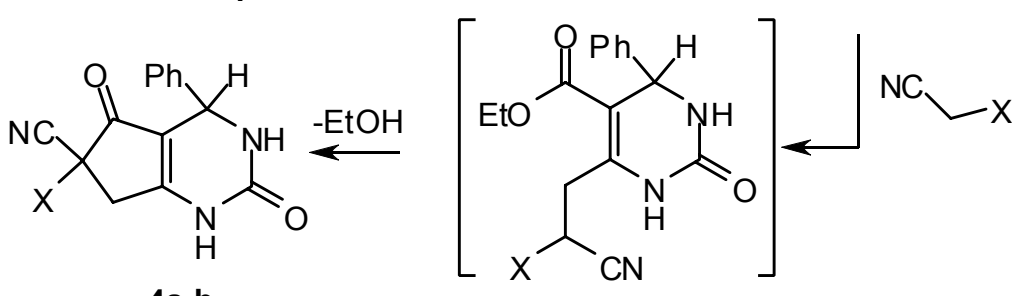

$4 a, b$

3,4 a, $X=C N$

$3 a, b$

b, $X=$ COOEt

\section{Scheme 1}


6-Bromomethylpyrimidine 2 underwent nucleophilic substitution reaction upon treatment with potassium cyanide to afford ethyl 6-cyanomethyl-2-oxo-4-phenyl-1,2,3,4tetrahydropyrimidine-5-carboxylate (5). The latter product couples smoothly with 4chlorobenzenediazonium chloride to afford the corresponding hydrazone 6 (Scheme 2).

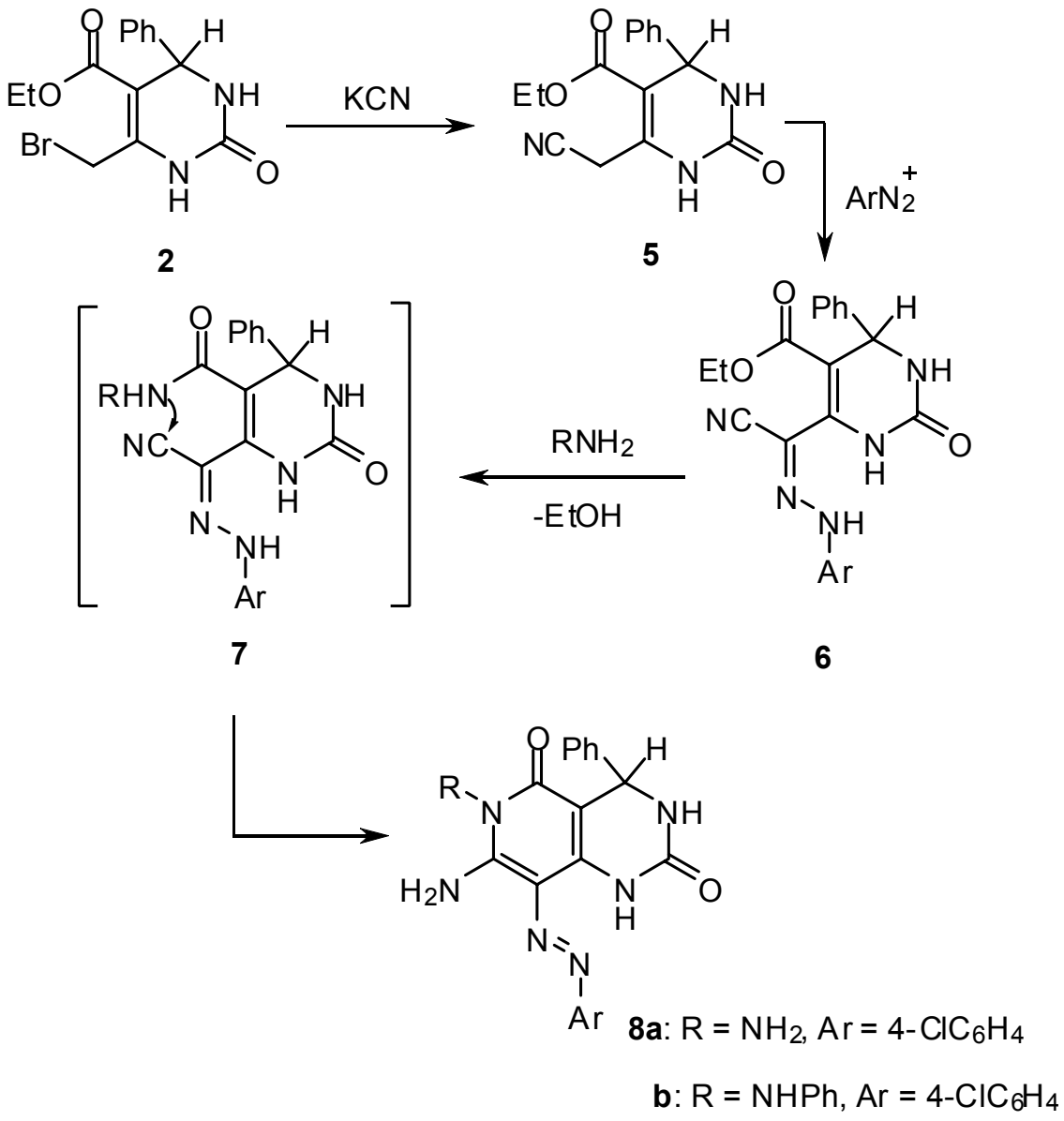

\section{Scheme 2}

The structures of compounds $\mathbf{5}$ and $\mathbf{6}$ were characterized from their spectroscopic as well as elemental analytical data. Thus, the IR spectrum of compound $\mathbf{5}$ revealed absorption bands at 3230, 3190, 2150, 1730 and $1645 \mathrm{~cm}^{-1}$ corresponding to two $\mathrm{C}=\mathrm{O}$ groups, $\mathrm{C} \equiv \mathrm{N}$ and two $\mathrm{NH}$ functions, respectively. Its ${ }^{1} \mathrm{H}$ NMR spectrum showed a triplet signal at $\delta 1.10(J=7.2 \mathrm{~Hz})$ corresponding to $\mathrm{CH}_{3}$ protons, a quartet signal at $\delta 4.00(J=7.2 \mathrm{~Hz})$ due to $\mathrm{CH}_{2}$ protons, a singlet signal at $\delta 4.25$ due to $\mathrm{CH}_{2}$ protons, a doublet signal at $\delta 5.22(J=3.3 \mathrm{~Hz})$ corresponding to $\mathrm{CH}-4$ proton, an aromatic multiplet in the region 7.24-7.35, and two $\mathrm{D}_{2} \mathrm{O}$-exchangeable signals at $\delta 7.55$ and 9.05 due two NH protons. The IR spectrum of compound 6 showed absorption bands at $1630,1690,2206,3094,3225$ and $3271 \mathrm{~cm}^{-1}$ corresponding to two $\mathrm{C}=\mathrm{O}$ groups, $\mathrm{C} \equiv \mathrm{N}$ and three $\mathrm{NH}$ functions, respectively. The ${ }^{1} \mathrm{H} \mathrm{NMR}$ spectrum of the same 
compound reveald a triplet signal at $\delta 1.09(J=7.2 \mathrm{~Hz})$ due to $\mathrm{CH}_{3}$ protons, a quartet signal at $\delta$ $4.06(J=7.2 \mathrm{~Hz})$ due to $\mathrm{CH}_{2}$ protons, and a doublet signal at $\delta 5.23(\mathrm{~J}=3.3 \mathrm{~Hz})$ due to the $\mathrm{CH}-$ 4 proton. It showed also three $\mathrm{D}_{2} \mathrm{O}$-exchangeable signals at $\delta 7.91,9.46$ and 11.60 due to three $\mathrm{NH}$ protons, in addition to an aromatic multiplet in the region 7.28-7.53.

When the hydrazone 6 was treated with hydrazine hydrate or with phenylhydrazine, it afforded the corresponding pyrido[4,3- $d]$ pyrimidine derivatives $\mathbf{8 a}$ and $\mathbf{8 b}$, respectively (Scheme 2 ). The structures of the products $\mathbf{8 a}$ and $\mathbf{8 b}$ were characterized from their spectroscopic as well as elemental analytical data. For example, the IR spectrum of compound 8a revealed the absence of an absorption band corresponding to the $\mathrm{C} \equiv \mathrm{N}$ function and showed absorption bands at 1659 , $1690,3105-3410 \mathrm{~cm}^{-1}$ corresponding to two $\mathrm{C}=\mathrm{O}, \mathrm{NH}_{2}$ and two $\mathrm{NH}$ functions, respectively. Its ${ }^{1} \mathrm{H}$ NMR spectrum revealed the absence of signals corresponding to $\mathrm{CH}_{3}$ and $\mathrm{CH}_{2}$ protons of the ethoxy group and showed a singlet signal at $\delta 5.46$ due to the $\mathrm{CH}$ proton and three $\mathrm{D}_{2} \mathrm{O}$ exchangeable signals at $\delta 5.77,7.80$ and 11.0 corresponding to $\mathrm{NH}_{2}$ and two $\mathrm{NH}$ protons, respectively, in addition to an aromatic multiplet and $\mathrm{NH}_{2}$ in the region $\delta$ 7.29-7.62.

The behavior of compound $\mathbf{2}$ towards phenylhydrazine as a nitrogen nucleophile was also investigated. Thus, when 2 was treated with phenylhydrazine, it afforded 4,6-diphenyl-3,4,7,8tetrahydropyrimido[4,5- $d$ ]pyridazine-2,5-(1H,6H)-dione (12) (Scheme 3). The structure of the isolated product 12 was evidenced by its spectroscopic and elemental analysis data.Thus, its IR spectrum exhibited absorption bands at 1665, 1687, 3010, 3120 and $3255 \mathrm{~cm}^{-1}$ corresponding to two $\mathrm{C}=\mathrm{O}$ groups, and three $\mathrm{NH}$ functions, respectively. Moreover, its ${ }^{1} \mathrm{H} \mathrm{NMR}$ spectrum revealed the absence of signals corresponding to $\mathrm{CH}_{3}$ and $\mathrm{CH}_{2}$ protons of an ethoxy group and revealed signals at $\delta 4.38$ and 5.19 due to $\mathrm{CH}_{2}, \mathrm{CH}$ protons and three $\mathrm{D}_{2} \mathrm{O}$-exchangeable signals at $\delta 7.61,9.26$ and 9.55 corresponding to three $\mathrm{NH}$ protons, in addition to an aromatic multiplet in the region $\delta 6.88-7.22$. 


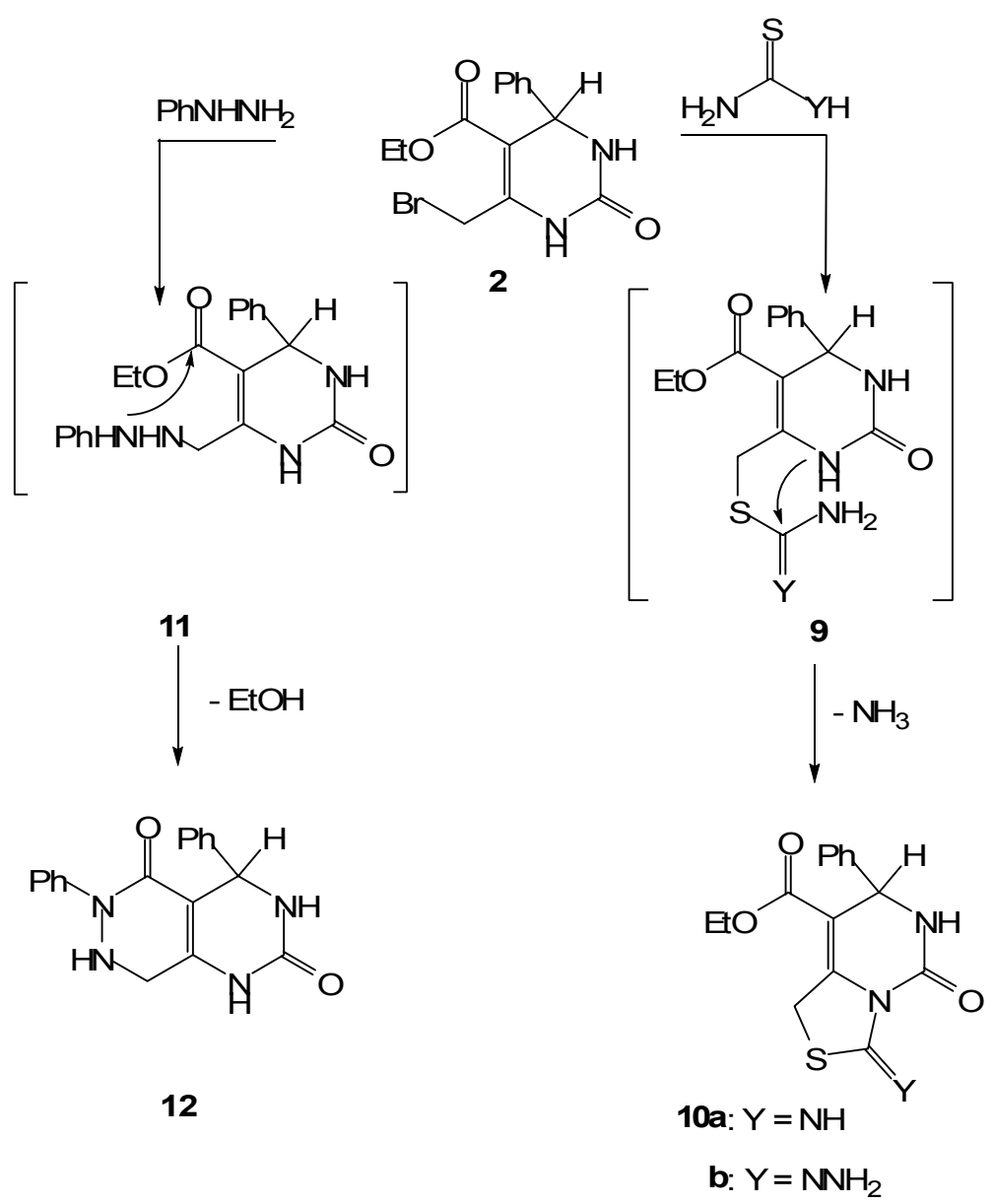

Scheme 3

\section{Conclusions}

In conclusion, we have investigated the synthetic potency of 6-(bromomethyl)-2-oxo-4-phenyl1,2,3,4-tetrahydropyrimidine-5-carboxylate (2) as a versatile, readily accessible building block for the synthesis of new fused heterocyclic compounds of biological and pharmaceutical importance.

\section{Experimental Section}

General Procedures. All melting points were measured with a Gallenkamp apparatus. The IR spectra were recorded of samples in $\mathrm{KBr}$ on a Shimadzu FT-IR 8101 PC infrared spectrophotometer. ${ }^{1} \mathrm{H}$ spectra were run at $300 \mathrm{MHz}$ and ${ }^{13} \mathrm{C}$ spectra were run at $75.46 \mathrm{MHz}$ in dimethyl sulphoxide (DMSO- $\left.d_{6}\right)$. Chemical shifts were related to that of the solvent. Mass 
spectra were measured on a GCMS-QP1000 EX spectrometer at $70 \mathrm{eV}$. Elemental analyses were carried out at the Microanalytical Center of Cairo University, Giza, Egypt. The biological evaluation of the products $\mathbf{4 a}, \mathbf{8 a}, \mathbf{8 b}$ and 12b were carried out at the Medical Mycology Laboratory of the Regional Center for Mycology and Biotechnology of Al-Azhar University, Cairo, Egypt. Ethyl 6-methyl-2-oxo-4-phenyl-1,2,3,4-tetrahydropyrimidine-5-carboxylate (1), ${ }^{19}$ ethyl 6-bromomethyl-2-oxo-4-phenyl-1,2,3,4-tetrahydropyrimidine-5-carboxylate (2) ${ }^{18}$ were prepared following the literature procedures.

Reaction of 6-bromomethylpyrimidine 2 with malononitrile and with ethyl acetoacetate. General procedure

To an ethanolic solution of 6-bromomethylpyrimidine $2^{18}(0.34 \mathrm{~g}, 1 \mathrm{mmol})$ and malononitrile $(0.066 \mathrm{~g}, 1 \mathrm{mmol})$ or with ethyl cyanoacetate $(0.113 \mathrm{~mL}, 1 \mathrm{mmol})$ was added few drops of piperidine and the reaction mixture was refluxed for $4 \mathrm{~h}$. The solid product was collected by filtration, washed with ethanol and purified by crystallisation from DMF to afford the corresponding cyclopenta $[d]$ pyrimidine derivatives $\mathbf{4 a}$ and $\mathbf{4 b}$, respectively.

\section{2,5-Dioxo-4-phenyl-3,4,5,7-tetrahydro-1- $H$-cyclopenta[d]pyrimidine-6,6(2H)-dicarbonitrile} (4a). Yield (70.21\%); mp. 290-1 ${ }^{\circ} \mathrm{C}$; IR (KBr) v $3350(\mathrm{NH}), 3102(\mathrm{NH}), 2210(\mathrm{C} \equiv \mathrm{N}), 2195$ $(\mathrm{C} \equiv \mathrm{N}), 1696(\mathrm{C}=\mathrm{O}), 1650(\mathrm{C}=\mathrm{O}) \mathrm{cm}^{-1} ;{ }^{1} \mathrm{H}$ NMR $\left(\mathrm{DMSO}-d_{6}\right) \delta 4.82\left(\mathrm{~s}, 2 \mathrm{H}, \mathrm{CH}_{2}\right), 5.25(\mathrm{~d}, 1 \mathrm{H}$, $\mathrm{CH}, J=3.3 \mathrm{~Hz}$ ), 7.26-7.39 (m, 5H, ArH's), 7.75 (s, 1H, $\mathrm{D}_{2} \mathrm{O}$-exchangeable, NH), 9.97 (s, 1H, $\mathrm{D}_{2} \mathrm{O}$-exchangeable, $\mathrm{NH}$ ). For $\mathrm{C}_{15} \mathrm{H}_{10} \mathrm{O}_{2} \mathrm{~N}_{4}$ Calcd.: $\mathrm{C}, 64.74 ; \mathrm{H}, 3.62 ; \mathrm{N}, 20.13$. Found: $\mathrm{C}, 64.65$; H, 3.67; N, 20.08\%.

Ethyl 6-cyano-2,5-dioxo-4-phenyl-2,3,4,5,6,7-hexahydro-1 $H$-cyclopenta[d]pyrimidine-6carboxylate (4b). Yield (69.87\%); mp. 285-6 ${ }^{\circ} \mathrm{C}$; IR (KBr) v $3312(\mathrm{NH}), 3220(\mathrm{NH}), 2210$ $(\mathrm{C} \equiv \mathrm{N}), 1690(\mathrm{C}=\mathrm{O}), 1635(\mathrm{C}=\mathrm{O}) \mathrm{cm}^{-1} ;{ }^{1} \mathrm{H}$ NMR $\left(\mathrm{DMSO}-d_{6}\right) \delta 1.14\left(\mathrm{t}, 3 \mathrm{H}, \mathrm{CH}_{3}, J=7.2 \mathrm{~Hz}\right), 4.1$ (q, $\left.2 \mathrm{H}, \mathrm{CH}_{2} J=7.2 \mathrm{~Hz}\right), 4.68\left(\mathrm{~s}, 2 \mathrm{H}, \mathrm{CH}_{2}\right), 5.24(\mathrm{~d}, 1 \mathrm{H}, \mathrm{CH}, J=3.3 \mathrm{~Hz}), 7.32-7.41(\mathrm{~m}, 5 \mathrm{H}$, ArH's), 7.73 (s, 1H, D $2 \mathrm{O}$-exchangeable, NH), 9.88 (s, 1H, $\mathrm{D}_{2} \mathrm{O}$-exchangeable, $\left.\mathrm{NH}\right)$. For $\mathrm{C}_{17} \mathrm{H}_{15} \mathrm{O}_{4} \mathrm{~N}_{3}$ Calcd.: C, 62.76; H, 4.65; N, 12.92. Found: C, 62.72; H, 4.68; N, 12.92\%.

\section{Ethyl 6-cyanomethyl-2-oxo-4-phenyl-1,2,3,4-tetrahydropyrimidine-5-carboxylate (5)}

A mixture of 6-bromomethylpyrimidine $2(0.34 \mathrm{~g}, 1 \mathrm{mmol})$ in EtOH $(10 \mathrm{~mL})$ and potassium cyanide $(0.65 \mathrm{~g}, 1 \mathrm{mmol})$ in water $(1 \mathrm{~mL})$ was heated under reflux for $1 \mathrm{~h}$ then left to cool. The reaction mixture was poured onto ice-cold water $(30 \mathrm{~mL})$. The solid precipitate was filtered off, washed with water and dried. Recrystallization from EtOH/water afforded the cyanomethyl derivative 5 in $60 \%$ yield, mp. $170{ }^{\circ} \mathrm{C}$; IR $(\mathrm{KBr}) \vee 3230(\mathrm{NH}), 3190(\mathrm{NH}), 2150(\mathrm{C} \equiv \mathrm{N}), 1730$ $(\mathrm{C}=\mathrm{O}), 1645(\mathrm{C}=\mathrm{O}) \mathrm{cm}^{-1} ;{ }^{1} \mathrm{H}$ NMR $\left(\mathrm{DMSO}_{-} d_{6}\right) \delta 1.1\left(\mathrm{t}, 3 \mathrm{H}, \mathrm{CH}_{3}, J=7.2 \mathrm{~Hz}\right), 4.0\left(\mathrm{q}, 2 \mathrm{H}, \mathrm{CH}_{2}\right.$, $J=7.2 \mathrm{~Hz}$ ), 4.25 (s, 2H, $\left.\mathrm{CH}_{2}\right), 5.22(\mathrm{~d}, 1 \mathrm{H}, \mathrm{CH}, J=3.3 \mathrm{~Hz}), 7.24-7.35$ (m, 5H, ArH's), 7.55 (s, $1 \mathrm{H}, \mathrm{D}_{2} \mathrm{O}$-exchangeable, $\left.\mathrm{NH}\right), 9.05$ (s, $1 \mathrm{H}, \mathrm{D}_{2} \mathrm{O}$-exchangeable, $\left.\mathrm{NH}\right)$. For $\mathrm{C}_{15} \mathrm{H}_{15} \mathrm{O}_{3} \mathrm{~N}_{3}$ Calcd.: $\mathrm{C}$, 63.15; H, 5.30; N, 14.73. Found:C, 63.14; H, 5.29; N, 14.68\%. 
Synthesis of ethyl 6-[(2-(4-chlorophenyl)hydrazono)(cyano)methyl]-2-oxo-4-phenyl-1,2,3,4tetrahydropyrimidine-5-carboxylate (6)

To a cold solution of the cyanomethyl derivative $5(2.85 \mathrm{~g}, 10 \mathrm{mmol})$ in ethanol $(50 \mathrm{~mL})$ and sodium acetate trihydrate $(3 \mathrm{~g})$ was added an equimolar amount of the diazonium chloride of 4chloroaniline [prepared by diazotizing 4-chloroaniline $(1.45 \mathrm{~g}, 10 \mathrm{mmol})$ in hydrochloric acid (6 M, $3 \mathrm{~mL})$ with sodium nitrite solution $(0.7 \mathrm{~g}, 10 \mathrm{mmol})$ in $5 \mathrm{~mL}$ water]. The addition, was carried out portionwise with stirring at $0-5{ }^{\circ} \mathrm{C}$ over a period of $30 \mathrm{~min}$. After complete addition, the reaction mixture was stirred for further $4 \mathrm{~h}$ then kept in an ice chest for $12 \mathrm{~h}$ and finally diluted with water. The precipitated solid was collected by filtration, washed with water, dried and finally recrystallized from acetic acid to afford the hydrazone derivative 6 in $79 \%$ yield, mp. 240-1 ${ }^{\circ} \mathrm{C}$; IR $(\mathrm{KBr}) \vee 3271(\mathrm{NH}), 3225(\mathrm{NH}), 3094(\mathrm{NH}), 2206(\mathrm{C} \equiv \mathrm{N}), 1690(\mathrm{C}=\mathrm{O}), 1630(\mathrm{C}=\mathrm{O})$ $\mathrm{cm}^{-1} ;{ }^{1} \mathrm{H}$ NMR (DMSO-d $) ~ \delta 1.09$ (t, 3H, $\mathrm{CH}_{3}, J=7.2 \mathrm{~Hz}$ ), 4.06 (q, 2H, $\mathrm{CH}_{2}, J=7.2 \mathrm{~Hz}$ ), 5.23 (d, 1H, CH, J=3.3 Hz), 7.3-7.5 (m, 9H, ArH's), 7.9 (s, 1H, D $2 \mathrm{O}$-exchangeable, NH), 9.48 (s, $1 \mathrm{H}, \mathrm{D}_{2} \mathrm{O}$-exchangeable, $\mathrm{NH}$ ), $11.63\left(\mathrm{~s}, 1 \mathrm{H}, \mathrm{D}_{2} \mathrm{O}\right.$-exchangeable, $\mathrm{NH}$ ); ${ }^{13} \mathrm{C}$ NMR (DMSO-do) $\delta$ 13.59 , 53.81, 60.21, 102.49, 107.15, 111.05, 116.89, 121.12, 126.08, 127.68, 128.55, 129.03, 140.36, 141.48, 143.43, 151.90, 164.33. For $\mathrm{C}_{21} \mathrm{H}_{18} \mathrm{O}_{3} \mathrm{~N}_{5} \mathrm{Cl}$ Calcd.: C, 59.51; H, 4.28; N, 16.52 . Found: C, 59.48; H, 4.26; N, 16.49\%.

Synthesis of 6,7-Disubstituted-8-(4-chlorophenylazo)-4-phenyl-3,4-dihydropyrido- [4,3d]pyrimidine-2,5-(1H, $6 \mathrm{H})$-dione derivatives 8a,b. General procedure

To a solution of the hydrazone $6(0.85 \mathrm{~g}, 2 \mathrm{mmol})$ in EtOH $(20 \mathrm{~mL})$, hydrazine hydrate $(80 \%$, $0.2 \mathrm{~mL}, 2 \mathrm{mmol})$ or phenylhydrazine $(0.216 \mathrm{~g}, 2 \mathrm{mmol})$ was added and the reaction mixture was refluxed for $4 \mathrm{~h}$, then allowed to cool. The solid product that formed was filtered off, washed with EtOH, and dried. Recrystallization from DMF/EtOH, afforded the corresponding pyrido[4,3-d]pyrimidine derivatives $\mathbf{8 a}$ and $\mathbf{8 b}$, respectively.

6,7-Diamino-8-(4-chlorophenylazo)-4-phenyl-3,4-dihydropyrido[4,3-d]pyrimidine-2,5-(1H, 6H)-dione (8a). Yield (79\%); mp. > $300{ }^{\circ} \mathrm{C}$; IR (KBr) v 3410, 3233, 3170, $3105\left(\mathrm{NH}_{2}, 2 \mathrm{NH}\right)$, $1690(\mathrm{C}=\mathrm{O}), 1659(\mathrm{C}=\mathrm{O}) \mathrm{cm}^{-1}{ }^{1} \mathrm{H}$ NMR $\left(\mathrm{DMSO}-d_{6}\right) \delta 5.46(\mathrm{~d}, 1 \mathrm{H}, \mathrm{CH}, J=2.7 \mathrm{~Hz}), 5.77(\mathrm{~s}, 2 \mathrm{H}$, $\mathrm{D}_{2} \mathrm{O}$-exchangeable $\left.\mathrm{NH}_{2}\right), 7.29-7.37\left(\mathrm{~m}, 7 \mathrm{H}, \mathrm{ArH}+\mathrm{NH}_{2}\right), 7.46(\mathrm{~d}, 2 \mathrm{H}, J=8.7 \mathrm{~Hz}), 7.62(\mathrm{~d}, 2 \mathrm{H}, J$ $=8.7 \mathrm{~Hz}), 7.80\left(\mathrm{~s}, 1 \mathrm{H}, \mathrm{D}_{2} \mathrm{O}\right.$-exchangeable, $\left.\mathrm{NH}\right), 11.00\left(\mathrm{~s}, 1 \mathrm{H}, \mathrm{D}_{2} \mathrm{O}\right.$-exchangeable, $\left.\mathrm{NH}\right) ;{ }^{13} \mathrm{C}$ NMR $\left(\mathrm{DMSO}-d_{6}\right) \delta 52.53,111.60,126.34,127.22,127.71,127.86,128.32,128.56,132.16,137.97$, 139.60, 142.40, 143.32, 151.10, 156.93. For $\mathrm{C}_{19} \mathrm{H}_{16} \mathrm{O}_{2} \mathrm{~N}_{7} \mathrm{Cl}$ Calcd.: C, 55.68; H, 3.94; N, 23.92; Cl, 8.65. Found: C, 55.61; H, 3.97; N, 23.95; Cl, 8.63\%.

7-Amino-8-((4-chlorophenyl)azo)-4-phenyl-6-(phenylamino)-3,4-dihydropyrido[4,3-d]pyrimidine-2,5-(1H,6H)dione (8b). Yield (80\%); mp. 254-5 ${ }^{\circ} \mathrm{C}$; IR (KBr) v 3320, 3210, $3190(\mathrm{NH})$, $1665(\mathrm{C}=\mathrm{O}), 1625(\mathrm{C}=\mathrm{O}) \mathrm{cm}^{-1}$; ${ }^{1} \mathrm{H}$ NMR (DMSO-d $) \delta 5.49(\mathrm{~d}, 1 \mathrm{H}, \mathrm{CH}, J=2.4 \mathrm{~Hz}), 6.26(\mathrm{~s}, 2 \mathrm{H}$, $\mathrm{D}_{2} \mathrm{O}$-exchangeable $\left.\mathrm{NH}_{2}\right), 6.77(\mathrm{~m}, 1 \mathrm{H}), 6.96(\mathrm{~d}, 2 \mathrm{H}), 7.21-7.40(\mathrm{~m}, 7 \mathrm{H}), 7.48(\mathrm{~d}, 2 \mathrm{H}, J=8.4$ $\mathrm{Hz}), 7.64(\mathrm{~d}, 2 \mathrm{H}, J=8.4 \mathrm{~Hz}), 8.14\left(\mathrm{~s}, 1 \mathrm{H}, \mathrm{D}_{2} \mathrm{O}\right.$-exchangeable, $\left.\mathrm{NH}\right), 8.88\left(\mathrm{~s}, 1 \mathrm{H}, \mathrm{D}_{2} \mathrm{O}-\right.$ exchangeable, $\mathrm{NH}), \delta 10.91\left(\mathrm{~s}, 1 \mathrm{H}, \mathrm{D}_{2} \mathrm{O}\right.$-exchangeable, $\left.\mathrm{NH}\right) ;{ }^{13} \mathrm{C}$ NMR (DMSO- $\left.d_{6}\right) \delta 52.60$, $109.26,111.73$, 118.92, 126.42, 127.32, 127.46, 127.91, 128.34, 128.59, 129.26, 132.26, 137.78, 
139.58, 141.07, 142.30, 145.46, 151.05, 156.94. For $\mathrm{C}_{25} \mathrm{H}_{20} \mathrm{O}_{2} \mathrm{~N}_{7} \mathrm{Cl}$ Calcd.:C, 61.79; H, 4.15; N, 20.18; Cl, 7.30. Found:C, 61.72; H, 4.08; N, 20.13; Cl, 7.28\%.

\section{Ethyl 3-substituted-3,5,6,7-tetrahydro-5-oxo-7-phenyl-1H-thiazolo[3,4-c]pyrimidine-8-} carboxylate 10a,b. General procedure

To a solution of the 6-bromomethypyrimidine derivative $2(0.68 \mathrm{~g}, 2 \mathrm{mmol})$ in EtOH $(20 \mathrm{~mL})$, thiourea $(0.152 \mathrm{~g}, 2 \mathrm{mmol})$ or thiosemicarbazide $(0.184 \mathrm{~g}, 2 \mathrm{mmol})$ was added and the reaction mixture was refluxed for $4 \mathrm{~h}$, then allowed to cool. The solid product was collected by filtration, washed with EtOH and crystallised from EtOH to afford the corresponding thiazolo[3,4c]pyrimidine $10 \mathrm{a}$ and $\mathbf{1 0 b}$, respectively.

Ethyl 3-imino-5-oxo-7-phenyl-3,5,6,7-tetrahydro-1-H-thiazolo[3,4-c]pyrimidine-8-carboxylate (10a). Yield (59\%); mp.197-9 ${ }^{\circ} \mathrm{C}$; IR (KBr) $\vee 3215(\mathrm{NH}), 3190(\mathrm{NH}), 1720(\mathrm{C}=\mathrm{O}), 1670$ $(\mathrm{C}=\mathrm{O}) \mathrm{cm}^{-1} ;{ }^{1} \mathrm{H} \mathrm{NMR}\left(\mathrm{DMSO}-d_{6}\right) \delta 0.90\left(\mathrm{t}, 3 \mathrm{H}, \mathrm{CH}_{3}, J=7.2 \mathrm{~Hz}\right), 4.15\left(\mathrm{q}, 2 \mathrm{H}, \mathrm{CH}_{2}, J=7.2 \mathrm{~Hz}\right)$, 4.9 (s, 2H, $\left.\mathrm{CH}_{2}\right), 5.3$ (s, 1H, CH), 7.12-7.57 (m, 5H, ArH's), 9.35 (s, 1H, D $2 \mathrm{O}$-exchangeable, $\mathrm{NH}), 11.97$ (s, 1H, D $2 \mathrm{O}$-exchangeable, $\mathrm{NH})$. For $\mathrm{C}_{15} \mathrm{H}_{15} \mathrm{O}_{3} \mathrm{~N}_{3} \mathrm{~S}$ Calcd.: C, 56.77; H, 4.76; N, 13.24; S, 10.10. Found: C, 56.73; H, 4.80; N, 13.23; S, 10.06\%.

Ethyl 3-hydrazono-5-oxo-7-phenyl-3,5,6,7-tetrahydro-1-H-thiazolo[3,4-c]pyrimidine-8carboxylate (10b). Yield (66\%); mp. $192{ }^{\circ} \mathrm{C}$; IR (KBr) v $3285(\mathrm{NH}), 3205-3175\left(\mathrm{NH}_{2}\right), 1720$ $(\mathrm{C}=\mathrm{O}), 1670(\mathrm{C}=\mathrm{O}) \mathrm{cm}^{-1} ;{ }^{1} \mathrm{H}$ NMR $\left(\mathrm{DMSO}_{-} d_{6}\right) \delta 0.97\left(\mathrm{t}, 3 \mathrm{H}, \mathrm{CH}_{3}, J=7.2 \mathrm{~Hz}\right), 4.18\left(\mathrm{q}, 2 \mathrm{H}, \mathrm{CH}_{2}\right.$, $J=7.2 \mathrm{~Hz}), 4.50\left(\mathrm{~s}, 2 \mathrm{H}, \mathrm{CH}_{2}\right), 5.14(\mathrm{~s}, 1 \mathrm{H}, \mathrm{CH}), 7.08-7.45$ (m, 5H, ArH's), 8.55 (s, 1H, D $\mathrm{O}_{-}$ exchangeable, $\mathrm{NH}_{2}$ ), $11.56\left(\mathrm{~s}, 1 \mathrm{H}, \mathrm{D}_{2} \mathrm{O}\right.$-exchangeable, $\mathrm{NH}$ ). For $\mathrm{C}_{15} \mathrm{H}_{16} \mathrm{O}_{3} \mathrm{~N}_{4} \mathrm{~S}$ Calcd.: $\mathrm{C}, 54.20$; H, 4.85; N: 16.86; S, 9.65. Found: C, 54.28; H, 4.88; N, 16.82; S, 9.63\%.

\section{4,6-Diphenyl-3,4,7,8-tetrahydropyrimido[4,5-d]pyridazine-2,5-(1H, 6H)-dione (12).}

To a solution of compound $2(0.68 \mathrm{~g}, 2 \mathrm{mmol})$ in EtOH $(20 \mathrm{~mL})$, phenylhydrazine $(0.216 \mathrm{~g}, 2$ mmol) was added and the reaction mixture was refluxed for $4 \mathrm{~h}$ then allowed to cool. The solid product was collected by filtration, washed with EtOH and crystallised from DMF/EtOH to afford 12 in 60\% yield; mp 275-7 ${ }^{\circ} \mathrm{C}$; IR (KBr) $\vee 3255(\mathrm{NH}), 3120(\mathrm{NH}), 3010(\mathrm{NH}), 1687$ $(\mathrm{C}=\mathrm{O}), 1665(\mathrm{C}=\mathrm{O}) \mathrm{cm}^{-1} .{ }^{1} \mathrm{H}$ NMR $\left(\mathrm{DMSO}_{-} d_{6}\right) \delta 4.38\left(\mathrm{~s}, 2 \mathrm{H}, \mathrm{CH}_{2}\right), 5.19(\mathrm{~s}, 1 \mathrm{H}, \mathrm{CH}), 6.88-7.22$ (m, 10H, ArH's), 7.61 (s, 1H, D $2 \mathrm{O}$-exchangeable, NH), 9.26 (s, 1H, $\mathrm{D}_{2} \mathrm{O}$-exchangeable, NH), $9.55\left(\mathrm{~s}, 1 \mathrm{H}, \mathrm{D}_{2} \mathrm{O}\right.$-exchangeable, $\left.\mathrm{NH}\right) ;{ }^{13} \mathrm{C} \mathrm{NMR}\left(\mathrm{DMSO}-d_{6}\right) \delta 49.73,51.90,99.53,116.23$, $121.58,126.31,127.19,128.06,128.78,143.34,143.49,150.41,151.30,165.48$. For $\mathrm{C}_{18} \mathrm{H}_{16} \mathrm{O}_{2} \mathrm{~N}_{4}$ Calcd.: C, 67.49; H, 5.03; N, 17.49. Found: C, 67.52; H, 5.00; N, 17.52\%.

\section{Antimicrobial Activity}

Compounds 4a, 8a, 8b and 12b were tested for their antimicrobial activities using four fungal species, namely Aspergillus fumigatus AF, Penicillium italicum PI, Syncephalastrum racemosum $S R$ and Candida albicans CA. Also, four bacteria species namely, Staphylcoccus aureus SA, Psedomo naaeruginosa PA, Bacillusubtilis BS and Escherichia coli EC were tested. The organisms were tested against the activity of solutions of concentration of $1 \mathrm{mg} / \mathrm{mL}, 2.5 \mathrm{mg} / \mathrm{mL}$ and $5 \mathrm{mg} / \mathrm{mL}$ of each compound and using an inhibition zone diameter in $\mathrm{cm}$ (IZD) as criterion 
for the antimicrobial activity. The fungicide Terbinafin and the bactericide Chloramphenicol were used as references to evaluate the potency of the tested compounds under the same conditions. The results are summarized in Table 1.

The test results revealed that all compounds exhibited a moderate activity against Staphylcoccus aureus (SA), Bacillus subtilis (BS) and Escherichia coli (EC) at concentrations of 5, 2.5 and 1 $\mathrm{mg} / \mathrm{mL}$. All compounds exhibited almost no activity against Aspergillus fumigatus (AF), Penicillium italicum (PI), Syncephalastrum racemosum (SR), Candida albicans (CA) and Pseudomonas aeruginosa (PA).

Table 1. Antimicrobial activity of compounds $4 \mathbf{a}, 8 \mathbf{8}, 8 \mathbf{b}$ and 12

\begin{tabular}{|c|c|c|c|c|c|c|c|c|c|c|c|c|c|c|c|}
\hline \multirow{2}{*}{$\begin{array}{l}\text { Sample } \\
\mathrm{mg} / \mathrm{ml}\end{array}$} & \multicolumn{3}{|c|}{$4 a$} & \multicolumn{3}{|c|}{$\mathbf{8 a}$} & \multicolumn{3}{|c|}{$8 b$} & \multicolumn{3}{|c|}{12} & \multicolumn{3}{|c|}{ Standard $\mathrm{mg} / \mathrm{mL}$} \\
\hline & 5 & 2.5 & 1 & 5 & 2.5 & 1 & 5 & 2.5 & 1 & 5 & 2.5 & 1 & 5 & 2.5 & 1 \\
\hline (AF) & 0 & 0 & 0 & 0 & 0 & 0 & 0 & 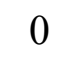 & 0 & 0 & 0 & 0 & 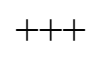 & . & ++ \\
\hline (PI) & 0 & 0 & 0 & 0 & 0 & 0 & 0 & 0 & 0 & 0 & 0 & 0 & +++ & +++ & ++ \\
\hline (SR) & 0 & 0 & 0 & 0 & 0 & 0 & 0 & 0 & 0 & 0 & 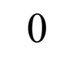 & 0 & +++ & +++ & +++ \\
\hline (CA) & 0 & 0 & 0 & 0 & 0 & 0 & 0 & 0 & 0 & 0 & 0 & 0 & ++ & ++ & ++ \\
\hline (SA) & ++ & ++ & ++ & ++ & ++ & ++ & ++ & ++ & ++ & ++ & ++ & ++ & ++ & ++ & ++ \\
\hline (PA) & 0 & 0 & 0 & 0 & 0 & 0 & 0 & 0 & 0 & 0 & 0 & 0 & +++ & +++ & ++ \\
\hline (BS) & ++ & ++ & ++ & ++ & ++ & ++ & ++ & ++ & ++ & ++ & ++ & ++ & +++ & +++ & ++ \\
\hline (EC) & ++ & ++ & ++ & ++ & ++ & ++ & ++ & ++ & ++ & ++ & ++ & ++ & ++ & ++ & ++ \\
\hline
\end{tabular}

Micro-organism/IZD (cm)*.

*IZD beyond control/(sign): 1.1-1.5 cm/(+++); 0.6-1.0 cm/(++); 0.1-0.5 cm/(+); $0 \mathrm{~cm} /(0)$.

\section{References}

1. Numazl, H.; Mirzeni, Y. R.; Azamat, H. J. Heterocycl. Chem. 2001, 38, 1051.

2. Haggarty, S. J.; Mayer, T. U.; Miyamoto, D. T.; Fathi, R;. King, R. W.; Mitchison, T. J.; Schreiber, S. L. Chem. Biol. 2000, 7, 275.

3. Kappe, C. O. Acc. Chem. Res. 2000, 33, 879.

4. Kappe, C. O. Eur. J. Med. Chem. 2000, 35, 1043.

5. Grover, G. J.; Dzwonczyk, S.; McMullen, D. M.; Normandin, D. E.; Parham, C. S.; Sleph, P. G.; Moreland, S. J. Cardiovasc. Pharm. 1995, 26, 289.

6. Mayer, T. U.; Kapoor, T. M.; Haggarty, S. J.; King, R. W.; Schreiber, S. L.; Mitchison, T. J. Science 1999, 286, 971.

7. Dawood, K. M.; Farag, A. M.; Khedr, N. A. ARKIVOC 2008, (xv), 166.

8. Kheder, N. A.; Mabkhot, Y. N.; Farag, A. M. Synth. Commun. 2008, 38, 3170.

9. Kheder, N. A.; Mabkhot, Y. N.; Farag, A. M. Heterocycles 2008, 75, 887. 
10. Farag, A. M.; Mayhoub, A. S.; Barakat, S. E.; Bayomi, A. H. Bioorg. Med. Chem. 2008, 16, 881.

11. Farag, A. M.; Mayhoub, A. S.; Barakat, S. E.; Bayomi, A. H. Bioorg. Med. Chem. 2008, 16, 4569

12. Shaaban, M. R.; Saleh,T. S.; Mayhoub, A. S.; Mansour, A.; Farag, A. M. Bioorg. Med. Chem. 2008, 16, 6344.

13. Girgis, A. S.; Mishriky, N.; Farag, A. M.; El-Eraky, W. I.; Farag, H. Eur. J. Med. Chem. 2008, 43, 1818.

14. Farag, A. M.; Elkholy, Y. M.; Ali, K. A. J. Heterocycl. Chem. 2008, 45, 279.

15. Kheder, N. A.; Mabkhot, Y. N.; Farag, A. M. Heterocycles 2008, 75, 2937.

16. Shaaban M. R.; Eldebss, T. M. A.; Darweesh, A. F.; Farag, A. M. J. Heterocycl. Chem. 2008, 45, 1739.

17. Shaaban, M. R.; Saleh, T. S.; Farag, A. M. Heterocycles 2009, 78, 151.

18. Zigeuner, G.; Hamberger, H.; Blaschke, H.; Sterk, H. Monatsh. Chem. 1966, 97, 1408.

19. Biginelli, P. Gazz. Chim. Ital. 1893, 23, 360. 